\title{
ELECTROSTATIC EFFECTS IN PNEUMATIC TRANSPORT OF GRANULAR MATERIALS
}

\section{ELDIN WEE CHUAN LIM}

Department of Chemical and Biomolecular Engineering, National University of Singapore, 4 Engineering Drive 4, Singapore 117576

chelwce@nus.edu.sg

\section{JUN YAO}

School of Energy Research, Xiamen University, 422 South Siming Rd, Siming District, Xiamen, Fujian, 361005, P. R. China

yaojun@xти.edu.cn

YANLIN ZHAO

Institute of Particle Science and Engineering, School of Process, Environmental and Materials Engineering, University of Leeds, Leeds LS2 9JT, United Kingdom

\begin{abstract}
The methodology of coupling the Discrete Element Method (DEM) with Computational Fluid Dynamics (CFD) was applied for computational studies of pneumatic transport of granular materials through vertical and horizontal pipes in the presence of electrostatic effects. The simulations showed that a thin layer of particles formed and remained adhered to the pipe walls during the pneumatic conveying process due to the effects of strong electrostatic forces of attraction towards the pipe walls. Particle concentrations were generally higher near the pipe walls than at the pipe centre resulting in the ring flow pattern observed in previous experimental studies. The close correspondence between particle velocity vectors and fluid drag force vectors was indicative of the importance of fluid drag forces in influencing particle behaviors. In contrast, the much weaker particle-particle electrostatic repulsion forces had negligible effects on particle behaviors within the system under all operating conditions considered. The electrostatic field strength developed during pneumatic conveying increased with decreasing flow rate due to increased amount of particle-wall collisions. Based on dynamic analyses of forces acting on individual particles, it may be concluded that electrostatic effects played a dominant role in influencing particle behaviors during pneumatic conveying at low flow rates while drag forces became more important at high flow rates.
\end{abstract}

Keywords: Pneumatic transport; electrostatics; discrete element method; numerical simulation.

\section{Introduction}

Triboelectrification is the process of charge acquisition by solid particulate materials during flows due to repeated collisions between the solid particles with surfaces of a different material. It is an important phenomenon that arises in various environmental and industrial processes especially those that involve bounded turbulent flows of solid particulate materials. In recent years, the behaviors of granular materials under the effects 
of electrostatics have been investigated by several researchers. For example, the behaviors of small, conductive particles $(<<1 \mathrm{~mm})$ in known electric fields generated using a pair of parallel plates with alternating or direct voltages applied have been studied. The clustering patterns of such granular materials have been observed to be dependent on the voltage applied ${ }^{1-3}$ as well as the humidity of the environment ${ }^{4}$. A similar phenomenon was observed by Yao et al. ${ }^{5}$ for larger particles with diameter $2.8 \mathrm{~mm}$ where three characteristic aggregation patterns referred to as clusters, half-ring and ring were observed to form during pneumatic conveying through a vertical pipe in the presence of electrostatic effects. Al-Adel et al. ${ }^{6}$ investigated the effect of static electrification on gassolid flows in vertical risers and captured qualitative features of riser flows: core-annular particle distribution, annular particle downflow at low riser gas velocities, and annular upflow at high gas velocities. Joseph and Klinzing ${ }^{7}$ demonstrated that choking of granular flow was caused by electrostatic effects in vertical pneumatic transport. On the other hand, electrostatic effects were dependent on a variety of factors such as the physical, chemical and electrical characteristics of the material used and ambient conditions. Smeltzer et al. ${ }^{8}$ performed tests in a pneumatic conveying system using glass beads and found that at constant loadings small particles exhibited greater electrostatic effects than large particles. Matsusaka et al. ${ }^{9}$ developed a formulation for the variation of granule charging caused by repeated impacts on a wall and employed the formulation to granule charging in granular flows where each granule carried a different amount of charge. They then analyzed theoretically the granule charge distribution. Recently, Yao and Wang ${ }^{10}$ developed a method to investigate the effects of granule size and shape on electrostatics generation in pneumatic conveying systems and demonstrated that the proposed method is a useful tool for characterization of electrostatic effects in systems where granules have different sizes and geometries. Numerical simulations may also provide a viable alternative to the study of electrostatic effects in granular flow systems. In the present study, the methodology of coupling CFD with the Discrete Element Method (DEM) was used to study pneumatic transport of granular materials through vertical and horizontal pipes in the presence of electrostatic effects.

\section{Mathematical Model}

\subsection{Discrete Element Method}

The methodology of DEM and its corresponding governing equations have been presented numerous times in the research literature ${ }^{11-15}$ and only a brief description will be presented here for sake of completeness. The method of implementation followed that used by Lim et al. ${ }^{16,17}$ in their numerical studies of pneumatic conveying of granular materials through vertical, horizontal and inclined pipes. The translational and rotational motions of individual solid particles are governed by Newton's laws of motion:

$$
m_{i} \frac{d v_{i}}{d t}=\sum_{j=1}^{N}\left(f_{c, i j}+f_{d, i j}\right)+m_{i} g+f_{f, i}+f_{e, i},
$$




$$
I_{i} \frac{d \omega_{i}}{d t}=\sum_{j=1}^{N} T_{i j},
$$

where $m_{i}$ and $v_{i}$ are the mass and velocity of $i^{\text {th }}$ particle respectively, $\mathrm{N}$ is the number of particles in contact with $\mathrm{i}^{\text {th }}$ particle, $\mathrm{f}_{\mathrm{c}, \mathrm{ij}}$ and $\mathrm{f}_{\mathrm{d}, \mathrm{ij}}$ are the contact and viscous contact damping forces respectively, $\mathrm{f}_{\mathrm{f}, \mathrm{i}}$ is the fluid drag due to the interstitial fluid respectively, $f_{e, i}$ is the electrostatic force, $I_{i}$ is the moment of inertia of $i^{\text {th }}$ particle, $\omega_{i}$ is its angular velocity and $\mathrm{T}_{\mathrm{ij}}$ is the torque arising from contact forces which causes the particle to rotate.

Contact and damping forces have to be calculated using force-displacement models that relate such forces to the relative positions, velocities and angular velocities of the colliding particles. Following previous studies ${ }^{11-17}$, a linear spring-and-dashpot model was implemented for the calculation of these collision forces. With such a closure, interparticle collisions are modeled as compressions of a perfectly elastic spring while the inelasticities associated with such collisions are modeled by the damping of energy in the dashpot component of the model. Collisions between particles and a wall may be handled in a similar manner but with the latter not incurring any change in its momentum. In other words, a wall at the point of contact with a particle may be treated as another particle but with an infinite amount of inertia. The normal $\left(f_{c n, i j}, f_{d n, i j}\right)$ and tangential $\left(f_{c t, i j}, f_{d t, i j}\right)$ components of the contact and damping forces are calculated according to the following equations:

$$
\begin{gathered}
f_{c n, i j}=-\left(\kappa_{n, i} \delta_{n, i j}\right) n_{i}, \\
f_{c t, i j}=-\left(\kappa_{t, i} \delta_{t, i j}\right) t_{i}, \\
f_{d n, i j}=-\eta_{n, i}\left(v_{r} \cdot n_{i}\right) n_{i}, \\
f_{d t, i j}=-\eta_{t, i}\left\{\left(v_{r} \cdot t_{i}\right) t_{i}+\left(\omega_{i} \times R_{i}-\omega_{j} \times R_{j}\right)\right\},
\end{gathered}
$$

where $\kappa_{n, i}, \delta_{n, i j}, n_{i}, \eta_{n, i}$ and $\kappa_{t, i}, \delta_{t, i j}, t_{i}, \eta_{t, i}$ are the spring constants, displacements between particles, unit vectors and viscous contact damping coefficients in the normal and tangential directions respectively, $v_{r}$ is the relative velocity between particles and $R_{i}$ and $R_{j}$ are the radii of particles $i$ and $j$ respectively. If $\left|f_{c t, i j}\right|>\left|f_{n t, i j}\right| \tan \phi$, then 'slippage' between two contacting surfaces is simulated based on Coulomb-type friction law, i.e. $\left|f_{c t, i j}\right|=\left|f_{n t, i j}\right| \tan \phi$, where tan $\phi$ is analogous to the coefficient of friction.

\subsection{Fluid Drag Force}

The fluid drag force model proposed by Di Felice ${ }^{18}$ which is applicable over a wide range of particle Reynolds numbers was used to evaluate the drag force. The equations in this fluid drag force model include:

$$
f_{f, i}=f_{f 0, i} \varepsilon_{i}^{-(\chi+1)},
$$




$$
\begin{gathered}
f_{f 0, i}=0.5 c_{d 0, i} \rho_{f} \pi R_{i}^{2} \varepsilon_{i}^{2}\left|u_{i}-v_{i}\right|\left(u_{i}-v_{i}\right), \\
\chi=3.7-0.65 \exp \left[-\frac{\left(1.5-\log _{10} \operatorname{Re}_{p, i}\right)^{2}}{2}\right], \\
c_{d 0, i}=\left(0.63+\frac{4.8}{\operatorname{Re}_{p, i}^{0.5}}\right)^{2}, \\
\operatorname{Re}_{p, i}=\frac{2 \rho_{f} R_{i} \varepsilon_{i}\left|u_{i}-v_{i}\right|}{\mu_{f}},
\end{gathered}
$$

where $\mathrm{f}_{\mathrm{f} 0, \mathrm{i}}$ is the fluid drag force on particle $\mathrm{i}$ in the absence of other particles, $\chi$ is an empirical parameter, $\varepsilon_{\mathrm{i}}$ is the local average porosity in the vicinity of particle $\mathrm{i}, \mathrm{c}_{\mathrm{d} 0, \mathrm{i}}$ is the drag coefficient, $\mathrm{Re}_{\mathrm{p}, \mathrm{i}}$ is the Reynolds number based on particle diameter, $\rho_{\mathrm{f}}$ is the fluid density, $\mu_{\mathrm{f}}$ is the fluid viscosity and $\mathrm{u}_{\mathrm{i}}$ is the fluid velocity.

\subsection{Computational Fluid Dynamics}

The motion of the continuum liquid phase is governed by the Navier-Stokes equations with interphase interactions taken into account as an additional source term in the momentum equation. The computational domain was divided into uniform $4 \mathrm{~mm} \times 4 \mathrm{~mm}$ computational cells and all quantities such as velocities and pressure were assumed constant over each cell. According to the local averaging technique, the continuity and momentum equations are expressed in terms of local mean variables over each computational cell:

$$
\begin{gathered}
\frac{\partial \varepsilon}{\partial t}+\nabla \cdot(\varepsilon u)=0 \\
\frac{\partial\left(\rho_{f} \varepsilon u\right)}{\partial t}+\nabla \cdot\left(\rho_{f} \varepsilon u u\right)=-\varepsilon \nabla P+\nabla \cdot\left(\mu_{f} \varepsilon \nabla u\right)+\rho_{f} \varepsilon g-F,
\end{gathered}
$$

where $\mathrm{u}$ is the velocity vector, $\varepsilon$ is the local average porosity, $\mathrm{P}$ is the fluid pressure and $\mathrm{F}$ is the source term due to fluid-particle interaction.

The source term F for a particular computational cell was calculated by summing the fluid drag forces on all particles present within that cell:

$$
F=\frac{\sum_{i=1}^{n} f_{f, i}}{D V},
$$

where $\Delta \mathrm{V}$ is the volume of a computational cell and $\mathrm{n}$ is the number of particles present in the cell. 


\subsection{Electrostatic Effects}

During pneumatic conveying, solid particles gain electrostatic charges as a result of repeated collisions and impacts against other particles and with the walls of the conveying pipe. The amount of charge transferred between two contacting bodies during impact depends on the potential difference between the two bodies, which in turn depends on their surface work functions and the image charge effect. In addition, the large group of charged particles within a conveying line gives rise to an electric field that can also influence the total potential difference through what is known as the space charge effect. It may be expected that a comprehensive mathematical model which incorporates such effects would be necessary for a detailed transient simulation of the electrification of particles starting from uncharged ones. Such a model is beyond the scope of the present study. Instead, it was assumed that the pneumatic conveying system had been operated beyond the transient state of electrification such as to reach a dynamic state of electrostatic equilibrium ${ }^{19}$. The amount of charge carried by the particles as well as the walls of the pipe then remained fairly constant with respect to time. In general, charges are transferred between particles and the pipe walls during particle-wall collisions due to differences in material properties between the two contacting bodies while charge transfer between two colliding particles may occur due to differences in electrical potential or charge densities. At the state of electrostatic equilibrium, it may be expected that particles and the pipe walls are oppositely charged relatively to each other while all particles carry the same type of charges. In other words, attractive electrostatic forces exist between particles and pipe walls while repulsive electrostatic forces exist between any pair of particles. The total electrostatic force acting on each particle may then be written as the sum of electrostatic forces due to charges carried by other particles and the pipe walls.

$$
f_{e, i}=f_{e p, i}+f_{e w, i},
$$

where $f_{e p, i}$ and $f_{e w, i}$ are the electrostatic forces due to other charged particles and the pipe walls acting on particle i respectively. It may also be mentioned at this point that at the state of electrostatic equilibrium, the linear charge density of the pipe walls is assumed to give rise to electrostatic forces of attraction acting on particles near the walls that are much more significant than image charge forces. In other words, it is assumed that image charge forces are negligible in comparison with the electrostatic forces due to charged pipe walls when the state of electrostatic equilibrium has been attained.

The electrostatic force arising from charges carried by other particles may be calculated by assuming each particle to be a constant point charge.

$$
f_{e p, i}=\sum_{\substack{j=1 \\ j^{\prime} i}}^{N} \frac{Q^{2}}{4 p e_{o} r_{i j}^{2}} n_{i},
$$


where $\mathrm{Q}$ is the constant charge assumed to be carried by all particles, $\varepsilon_{\mathrm{o}}$ is the permittivity of free space, $r_{i j}$ is the distance between particles $i$ and $j$ and $n_{i}$ is the unit normal vector in the direction of the line joining the two particle centers.

The average electric field strength near a wall of the pneumatic conveying pipe may be estimated by assuming the pipe to be a flat plat infinitely long in the axial direction ${ }^{17}$.

$$
E=\int \frac{1}{4 \pi \varepsilon_{0}} \cdot \frac{d q}{r^{2}}=\frac{\lambda}{2 \pi \varepsilon_{0} d} \int_{0}^{\frac{\pi}{2}} \cos \theta d \theta=\frac{\lambda}{2 \pi \varepsilon_{0} d},
$$

where $\mathrm{E}$ is the electric field strength, $\mathrm{q}$ is the equilibrium charge on the pipe wall, $\varepsilon_{\mathrm{o}}$ is the permittivity of free space, $r$ is the average distance of particles to the pipe wall, $\lambda$ is the linear charge density along the pipe wall. The electrostatic force on a charged particle $\mathrm{i}$ located near the pipe wall may then be calculated as $\mathrm{f}_{\mathrm{ew}, \mathrm{i}}=\mathrm{E} \cdot \mathrm{Q}$.

\subsection{Simulation Conditions}

The geometry of the pneumatic conveying system and type of particles used in the present simulations are summarized in Tables 1 and 2. These were based on the experimental work of Yao et al. ${ }^{19}$ so that a meaningful comparison between the simulation and experimental outputs can be made. The overall solid fraction was about $5 \%$ as applied in the experimental studies of the previous researchers. In all simulations performed, particles were first allowed to settle freely under gravity for $0.5 \mathrm{~s}$ before gas flow was initiated. Periodic boundary conditions were applied to the solid phase to simulate an open flow system. Particles which were carried out of the conveying pipe by the flowing gas were simulated to re-enter from the inlet of the pipe with the same velocities and radial positions.

Table 1. Material properties and system parameters.

\begin{tabular}{ll}
\hline Shape of particles & Spherical \\
Particle diameter, $\mathrm{d}$ & $2.8 \mathrm{~mm}$ \\
Particle density, $\rho_{\mathrm{p}}$ & $1123 \mathrm{~kg} \mathrm{~m}^{-3}$ \\
Coefficient of restitution & 0.9 \\
Coefficient of friction & 0.3 \\
Gas density, $\rho_{\mathrm{f}}$ & $1.205 \mathrm{~kg} \mathrm{~m}^{-3}$ \\
Gas viscosity, $\mu_{\mathrm{f}}$ & $1.8 \times 10^{-5} \mathrm{~N} \mathrm{~s} \mathrm{~m}^{-2}$ \\
Pipe diameter & $0.04 \mathrm{~m}$ \\
Pipe length & $1.0 \mathrm{~m}$ \\
Computational cell size & $4 \mathrm{~mm} \times 4 \mathrm{~mm}$ \\
Simulation time step, $\Delta \mathrm{t}$ & $10^{-6}-10^{-5} \mathrm{~s}$ \\
\hline
\end{tabular}


Table 2. Electrostatic parameters for vertical pneumatic conveying simulations.

\begin{tabular}{ccc}
\hline $\begin{array}{c}\text { Inlet gas velocity } \\
\left(\mathrm{m} \mathrm{s}^{-1}\right)\end{array}$ & $\begin{array}{c}\text { Particle charge density } \\
\left(10^{-9} \mathrm{C} \mathrm{g}^{-1}\right)\end{array}$ & $\begin{array}{c}\text { Pipe wall linear charge } \\
\text { density } \lambda\left(\mathrm{C} \mathrm{m}^{-1}\right)\end{array}$ \\
\hline 12.6 & 2.36 & $-1.57 \times 10^{-5}$ \\
14.6 & 2.05 & $-3.27 \times 10^{-6}$ \\
21.2 & 1.22 & $-1.14 \times 10^{-6}$
\end{tabular}

\section{Results and Discussion}

Figure 1a shows an instantaneous snapshot of the pneumatic conveying process obtained from DEM simulations for an air flow rate of $950 \mathrm{~L} \mathrm{~min}^{-1}$. The state of electrostatic equilibrium was assumed to be established throughout the conveying process. It may be seen here that a thin layer of particles remained adhered to the pipe walls under the effects of strong electrostatic forces of attraction towards the pipe walls. Particle concentrations were generally higher near the pipe walls than at the pipe centre and such a flow pattern would correspond to the ring flow pattern observed in previous experimental studies. ${ }^{5}$ Figure $1 \mathrm{~b}$ shows that most particles in the conveying pipe moved in the axial direction. Particle velocities were generally higher near the pipe centre than at the pipe walls due to similar air velocity profiles developed over the cross section of the pipe. However, it may also be seen that most particles moved at much lower velocities than the average gas velocity of $12.6 \mathrm{~m} \mathrm{~s}^{-1}$. Figure $1 \mathrm{c}$ shows that fluid drag forces acting on particles were predominantly oriented in the axial direction, indicating that the direction of motion of particles was largely a result of fluid drag forces. Although electrostatic forces that arose due to particle-particle repulsion might have the effect of disrupting the almost unidirectional motion of particles, Figure 1d shows that such forces were much smaller in magnitudes than fluid drag forces and so did not exert significant effects on particle behaviors during the pneumatic conveying process. It may thus be concluded at this point that the ring flow pattern observed in experimental studies of pneumatic conveying through vertical pipes arose due to two predominant types of forces present within the system. Electrostatic forces of attraction resulted in the adherence of particles onto the pipe walls while fluid drag forces moved the particles along the pipe in the axial direction. These two aspects of solid pattern formation and solid motion had minimal disruptive effects on each other as the two types of forces that gave rise to them were oriented more or less normal to each other. In contrast, Figure 1e shows that particles were distributed fairly homogeneously in the radial direction during pneumatic conveying in the absence of any electrostatic effects. This is reminiscent of the dispersed flow pattern that had been observed in previous experimental studies where at the start of each pneumatic conveying experiment, the dispersed flow pattern was formed as the amount of electrostatic charges present on the particles as well as the pipe walls was negligible 5 . 


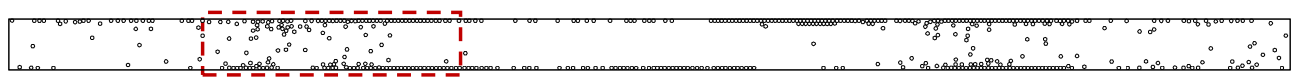

(a)

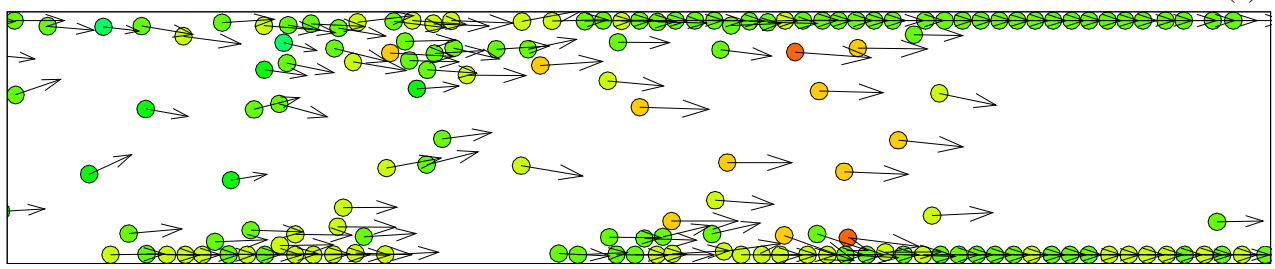

(b)

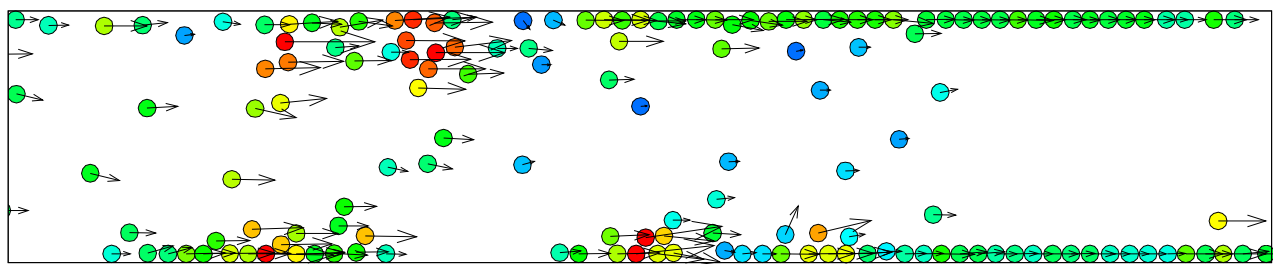

(c)

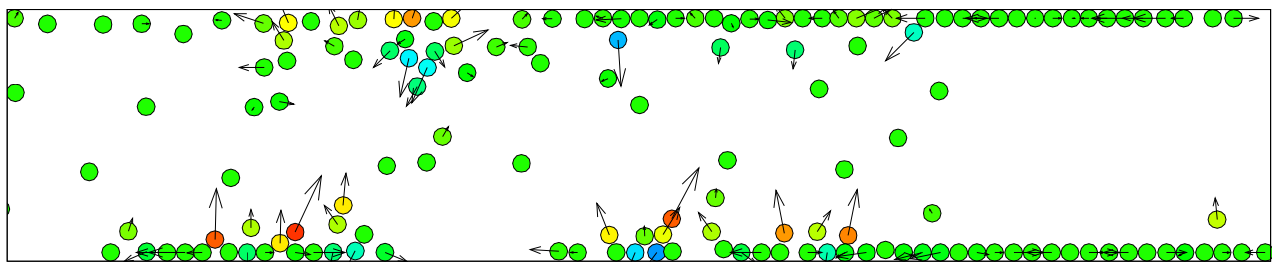

(d)

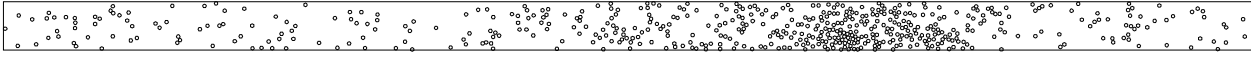

(e)

Fig. 1. (a) Instantaneous particle distribution during vertical pneumatic conveying at an air flow rate of $950 \mathrm{~L}$ $\mathrm{min}^{-1}$ in the presence of electrostatic effects. The region marked by a dashed box is enlarged in the next three panels. (b) Instantaneous particle velocity vectors with color contours indicating magnitudes of axial components of particle velocities ranging linearly from $0 \mathrm{~m} \mathrm{~s}^{-1}$ (blue) to $12.6 \mathrm{~m} \mathrm{~s}^{-1}$ (red). (c) Instantaneous fluid drag force vectors with color contours indicating magnitudes of axial components of fluid drag forces ranging linearly from $0 \mathrm{~N}$ (blue) to $2.0 \times 10^{-4} \mathrm{~N}$ (red). (d) Instantaneous interparticle electrostatic repulsion force vectors with color contours indicating radial components of interparticle electrostatic repulsion forces ranging linearly from $-10^{-6} \mathrm{~N}$ (blue) to $10^{-6} \mathrm{~N}$ (red). (e) Instantaneous particle distribution during vertical pneumatic conveying at an air flow rate of $950 \mathrm{~L} \mathrm{~min}^{-1}$ in the absence of electrostatic effects. The direction of gravity is towards the left of each panel.

The flow pattern observed at a higher air flow rate of $1100 \mathrm{~L} \mathrm{~min}^{-1}$ was similar. Due to the slightly weaker electrostatic field present, a thinner layer of particles was seen adhered to most parts of the pipe walls. In addition, there was a tendency for particles to move in the form of clusters near the pipe centre. At the highest air flow rate of $1600 \mathrm{~L}$ $\mathrm{min}^{-1}$ considered in this study, the flow pattern obtained from the DEM simulations shows that the characteristic ring flow pattern was no longer easily discernable due to much fewer particles remaining adhered to the pipe walls during the conveying process. 
This was a direct consequence of the weak electrostatic field present under such flow conditions.

The above computational methodology was subsequently applied towards studies of horizontal pneumatic conveying. The electrostatic parameters applied here were also based on the experimental work of Yao et al. ${ }^{19}$ and are shown in Table 3. On comparison with Table 2, it may be observed that particle charge densities developed during horizontal pneumatic conveying were approximately one order of magnitude smaller than those for vertical pneumatic conveying at corresponding air flow rates. As such, electrostatic effects were expected to play a less significant role in affecting solid flow patterns in the present case.

Table 3. Electrostatic parameters for horizontal pneumatic conveying simulations

\begin{tabular}{ccc}
\hline $\begin{array}{c}\text { Inlet gas velocity } \\
\left(\mathrm{m} \mathrm{s}^{-1}\right)\end{array}$ & $\begin{array}{c}\text { Particle charge density } \\
\left(10^{-9} \mathrm{C} \mathrm{g}^{-1}\right)\end{array}$ & $\begin{array}{c}\text { Pipe wall linear charge } \\
\text { density } \lambda\left(\mathrm{C} \mathrm{m}^{-1}\right)\end{array}$ \\
\hline 12.6 & 2.36 & $-1.62 \times 10^{-6}$ \\
14.6 & 2.05 & $-8.89 \times 10^{-7}$ \\
21.2 & 1.22 & $-1.32 \times 10^{-7}$ \\
\hline
\end{tabular}

Figure 2a shows an instantaneous snapshot of the horizontal pneumatic conveying process for an air flow rate of $950 \mathrm{~L} \mathrm{~min}^{-1}$. It may be seen that particles in this case were asymmetrically distributed about the axis of the pipe due to the effect of gravitational settling. Fluid drag forces were oriented predominantly in the axial direction (Figure 2b) while particle-particle electrostatic repulsion forces were oriented randomly (Figure 2c). As in the case of vertical pneumatic conveying, the latter was much smaller in magnitude and so did not lead to dispersion of the particles. Figure $2 \mathrm{~d}$ shows an instantaneous snapshot of the moving dunes flow pattern typically observed in horizontal pneumatic conveying at low solid concentrations in the absence of electrostatic effects. Due to the much weaker electrostatic forces present during horizontal pneumatic conveying, the solid flow patterns developed at the state of electrostatic equilibrium were similar to those observed in the absence of any electrostatic effects. The above observations were also applicable to horizontal pneumatic conveying at air flow rates of $1100 \mathrm{~L} \mathrm{~min}^{-1}$ and $1600 \mathrm{~L} \mathrm{~min}^{-1}$ where electrostatic effects were even weaker.

\section{Conclusions}

Pneumatic transport of granular materials through vertical and horizontal pipes in the presence of electrostatic effects has been investigated computationally using Computational Fluid Dynamics (CFD) and the Discrete Element Method (DEM) in this study. For all cases considered in this study, electrostatic field strength was highest near the pipe walls and degraded towards the pipe centre. The electrostatic field strength was 


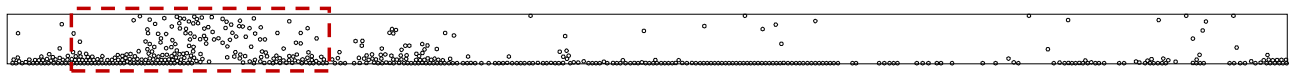

(a)

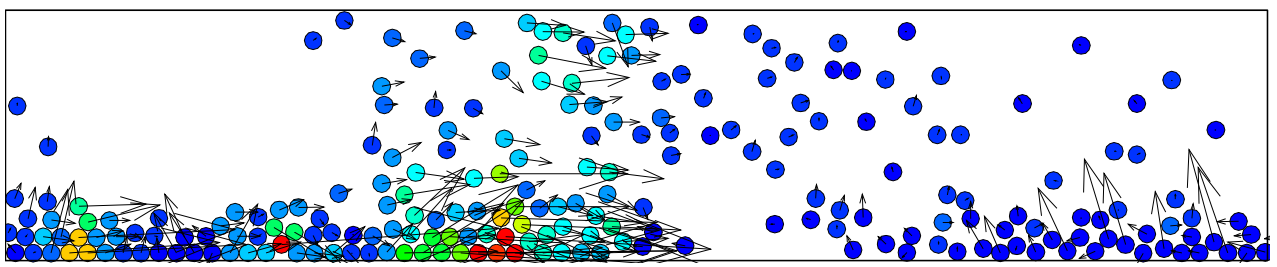

(b)

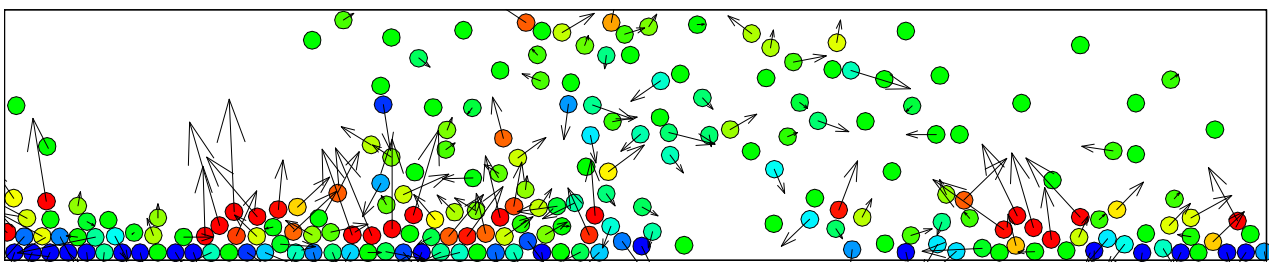

(c)

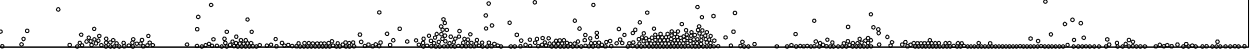

(d)

Fig. 2. (a) Instantaneous particle distribution during horizontal pneumatic conveying at an air flow rate of $950 \mathrm{~L}$ $\min ^{-1}$ in the presence of electrostatic effects. The region marked by a dashed box is enlarged in the next three panels. (b) Instantaneous fluid drag force vectors with color contours indicating magnitudes of axial components of fluid drag forces ranging linearly from $0 \mathrm{~N}$ (blue) to $1.0 \times 10^{-4} \mathrm{~N}$ (red). (c) Instantaneous interparticle electrostatic repulsion force vectors with color contours indicating radial components of interparticle electrostatic repulsion forces ranging linearly from $-10^{-6} \mathrm{~N}$ (blue) to $10^{-6} \mathrm{~N}$ (red). (d) Instantaneous particle distribution during horizontal pneumatic conveying at an air flow rate of $950 \mathrm{~L} \mathrm{~min}^{-1}$ in the absence of electrostatic effects. The direction of gravity is downwards.

also seen to increase with decreasing air flow rate. This may be explained by the larger number of particle-wall collisions that occurred and the larger number of particles that remained in contact with the pipe walls at any one instant in time during pneumatic conveying at lower flow rates than at higher flow rates. Typically, through the process of triboelectrification, all particles carry the same type of charges but are charged oppositely to the pipe walls when the state of electrostatic equilibrium has been attained. The DEM simulations showed that a thin layer of particles remained adhered to the pipe walls during the pneumatic conveying process due to the effects of strong electrostatic forces of attraction towards the pipe walls. Particle concentrations were generally higher near the pipe walls than at the pipe centre resulting in the ring flow pattern observed in previous experimental studies. The close correspondence between particle velocity vectors and fluid drag force vectors was indicative of the importance of fluid drag forces in influencing particle behaviors. In contrast, the much weaker particle-particle electrostatic repulsion forces had negligible effects on particle behaviors within the system under all operating conditions considered in this study. Based on dynamic analyses of forces acting on individual particles, it may be concluded that electrostatic effects played a dominant 
role in influencing particle behaviors during pneumatic conveying through vertical and horizontal pipes at low flow rates while drag forces became more important at high flow rates.

\section{Acknowledgments}

This study has been supported by the National University of Singapore under Grant No. R-279-000-275-112. The award of a Lee Kuan Yew Postdoctoral Fellowship to E.W.C. Lim by the National University of Singapore is gratefully acknowledged.

\section{References}

1. S. R. Yeh, M. Seul and B. I. Shralman, Nature, 386, 57 (1997).

2. M. V. Sapozhnikov, Y. V. Tolmachev, I. S. Aranson and W. K. Kwok, Phys. Rev. Lett. 90, 114301 (2003).

3. I. S. Aranson, D. Blair, V. A. Kalatsky, and et al., Phys. Rev. Lett. 84, 3306 (2000).

4. D. W. Howell, I. S. Aronson and G. W. Crabtree, Phys Rev E. 63, 050301 (2001).

5. J. Yao, Y. Zhang, C. H. Wang, and et al., Ind. Eng. Chem. Res. 43, 7181 (2004).

6. M. F. Al-Adel, D. A. Saville and S. Sundaresan, Ind. Eng. Chem. Res. 41, 6224 (2002).

7. S. Joseph and G. E. Klinzing, Powder Tech. 36, 79 (1983).

8. E. E. Smeltzer, M. L. Weaver and G. E. Klinzing, Powder Tech. 33, 31 (1982).

9. S. Matsusaka, T. Nishida, Y. Gotoh and H. Masuda, Adv. Powder. Tech. 14, 127 (2003).

10. J. Yao and C. H. Wang, Chem. Eng. Sci. 61, 3858 (2006).

11. E. W. C. Lim, Chem. Eng. Sci. 62, 4529 (2007).

12. E. W. C. Lim, Ind. Eng. Chem. Res. 47, 481 (2008).

13. E. W. C. Lim, Phys. Rev. E. 79, 041302 (2009).

14. E. W. C. Lim, AIChE. J. 56, 2588 (2010).

15. E. W. C. Lim, Eur. Phys. J. E. 32, 365 (2010).

16. E. W. C. Lim, C. H. Wang and A. B. Yu, AIChE. J. 52, 496 (2006).

17. E. W. C. Lim, Y. Zhang and C. H. Wang, Chem. Eng. Sci. 61, 7889 (2006).

18. R. Di Felice, Int. J. Multiphase Flow, 20, 153 (1994).

19. J. Yao, Y. Zhang, C. H. Wang and Y. C. Liang, AIChE. J. 52, 3775 (2006). 